\title{
Packet Replication and Noise in Reliable End-to-end Molecular Communication
}

\author{
Taro Furubayashi ${ }^{1}$, Tadashi Nakano ${ }^{1,2}$, Andrew Eckford ${ }^{3}$, \\ Norikazu Ichihashi ${ }^{4}$, Tetsuya Yomo ${ }^{1,4}$ \\ ${ }^{1}$ Graduate School of Frontier Biosciences, Osaka University, Japan \\ ${ }^{2}$ Institute of Academic Initiatives, Osaka University, Japan \\ ${ }^{3}$ Department of Electrical Engineering and Computer Science, York University, Canada \\ ${ }^{4}$ Graduate School of Information Science and Technology, Osaka University, Japan \\ \{fbayashi, tadasi.nakano\}@fbs.osaka-u.ac.jp \\ aeckford@yorku.ca, \{ichihashi, yomo\}@ist.osaka-u.ac.jp
}

\begin{abstract}
In modern communication systems, noise represents the source of errors that disturb useful information signals for communication. In biological systems, however, noise plays an important role in their function. In this position paper, we point out that, in designing and engineering molecular communication systems, noise may be removed as in traditional communication systems while it may also be exploited as in biological systems. We first show our prior work on reliable end-to-end molecular communication systems that incorporate packet replication to improve the reliability of molecular communication. We then discuss how the molecular communication systems may be extended based on the observation that biological systems utilize noise for their function.
\end{abstract}

\section{Keywords}

Molecular communication, RNA, self-replication, noise

\section{INTRODUCTION}

In molecular communication, bio-nanomachines communicate by propagating message-carrying information molecules in an aqueous environment [4]. Bio-nanomachines in molecular communication are assumed to be nano-to-micro scale devices composed of biomaterials, and capable of biochemical functions such as sensing biochemical conditions in their environment. Examples of bio-nanomachines include liposomes that store and release molecules and genetically engineered cells that perform logical operations.

One of the design issues in molecular communication is concerned with path loss and attenuation. Molecular communication often relies on Brownian motion to propagate information molecules between bio-nanomachines, and the concentration of information molecules, representing the signal strength, therefore decreases with distance. To overcome the path loss and attenuation issues, signals need to be amplified when they are transmitted, or while they propagate in the environment.

Our previous work shows a design of reliable end-to-end molecular communication that can overcome the path loss and attenuation issues [2]. The reliable end-to-end molecular communication uses molecular packets as information molecules, and performs packet replication to increase the number of their copies. Numerical examples are provided to show the improved reliability of molecular communication. In addition, empirical results are shown to demonstrate the feasibility that molecular packets can be implemented as RNA molecules.

This paper extends our previous work by considering the impact of noise that may introduce errors in packet replication. In modern communication systems, noise represents a source of errors that disturb useful information signals for communication. In biological systems, however, noise plays an important role in their function. In this paper, we point out that, in designing and engineering molecular communication systems, noise may be removed as in traditional communication systems, or it may be exploited as in biological systems.

The rest of the paper is organized as follows. We first discuss our prior work on reliable end-to-end molecular communication systems that incorporate packet replication to improve the reliability of molecular communication (Section 2). We then show how the molecular communication systems may be extended based on the observation that biological systems utilize noise for their function (Section 3).

\section{RELIABLE END-TO-END MOLECULAR COMMUNICATION}

Our initial design of reliable end-to-end molecular communication [2] includes source, intermediate and destination bionanomachines that exchange structured information molecules, called molecular packets. A molecular packet consists of a data payload and a header. A data payload contains molecular messages to be exchanged between source and destination bio-nanomachines. A header contains control infor- 


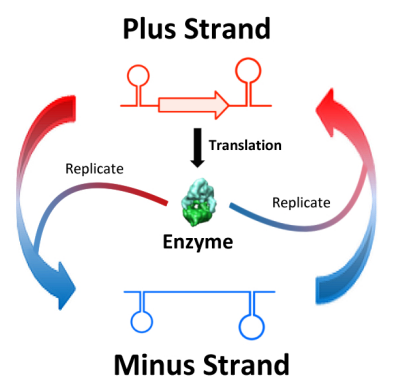

Figure 1: Molecular packet replication

mation such as a destination address to which the molecular packet is delivered, and an error handling code for the destination to detect and possibly correct errors contained in molecular packets.

The reliable end-to-end molecular communication consists of the following key processes: packet transmission for a source bio-nanomachine to encapsulate a molecular message into a molecular packet and transmit the molecular packet into the environment, packet propagation for the environment to propagate molecular packets, packet replication for an intermediate bio-nanomachine to selectively make copies of an incoming molecular packet, packet reception for a destination bio-nanomachine to extract a molecular message from an incoming molecular packet.

The reliable end-to-end molecular communication system is in part implemented based on an RNA self-replication system [3]. It consists of self-replicating RNA molecules and water solution containing all necessary materials for the RNA self-replication reaction as detailed below.

- A molecular packet is implemented as a self-replicating RNA molecule. The RNA molecule encodes an RNA replication enzyme in its sequence and self-replicates via translation of the RNA replication enzyme. Fig. 1 shows a schematic of the self-replication reaction. As shown in the figure, two types of RNA sequence are involved in the self-replication reaction: the proteinencoding "plus" strand and its complementary "minus" strand. The plus strand encodes the RNA replication enzyme and it is also used as a template to produce the minus strand, while the minus strand is only used as a template to produce the plus strand.

- Bio-nanomachines are implemented as water droplets in water-in-oil emulsion. Bio-nanomachines with the packet replication functionality are implemented as water droplets containing necessary materials for the RNA self-replication reaction such as amino acids, NTPs (nucleoside triphosphate), and functional proteins including ribosomes.

\section{DISCUSSION}

Packet replication can improve the reliability of molecular communication by producing a number of copies (i.e., by increasing the signal-to-noise ratio). However, molecular operations in nano-to-micro scale environments are susceptible to noise, and errors may be introduced in packet replication. Types of errors that can occur in packet replication are point mutation, insertion, and deletion. Point mutation is replacement of a base with another base at one point in the newly produced RNA sequence. Insertion is the addition of a base or bases in the newly produced RNA sequence. Deletion is the subtraction of a base or bases in the newly produced RNA sequence.

The impact of errors in packet replication can be quantified through information theory. Consider that an RNA sequence $X$ produces an RNA sequence $Y$ through the RNA self-replication reaction. Following the traditional model of communication, the self-replication reaction is a communication channel, with channel input $X$ and channel output $Y$. Given that RNA bases have four types (A, G, C, U), an RNA sequence of $N$ bases can represent $\log _{2} 4^{N}(=2 N)$ bits of information. The number of bits that can be transmitted from $X$ through the channel to $Y$ depends on errors that may occur in the channel. For simplicity, assume that only point mutation occurs, the probability of point mutation is $p$, and $p$ is independent of the location and base in a sequence. Then, the channel becomes symmetric, and the maximum number of bits that can be transfered, namely, capacity $C$ is given as $C=N(2-H(p))$ where $H(p)=-p \log _{2}(p / 3)-(1-p) \log _{2}(1-p)$ is the entropy function. Traditional coding schemes for modern communication systems may be used to approach the capacity.

Existing research in molecular communication aims at minimizing the impact of noise on information transmission. On the other hand, biological systems that are highly robust and plastic at the same time seem to exploit noise for their function, for example, to coordinate the system behavior or adapt to unpredictable environments [1]. Based on the observation into biological systems, we may need to rethink the design strategy of molecular communication systems that is to exploit noise to improve the robustness and plasticity of molecular communication systems.

\section{Acknowledgments}

This work was supported through the Program for Leading Graduate Schools by Japan Society of the Promotion of Science (JSPS).

\section{REFERENCES}

[1] A. Eldar and M. B. Elowitz. Functional roles for noise in genetic circuits. Nature, 467:167-173, 2010.

[2] T. Furubayashi, T. Nakano, A. Eckford, and T. Yomo. Reliable end-to-end molecular communication with packet replication and retransmission. In IEEE Global Communications Conference (GLOBECOM 2015), 2015.

[3] N. Ichihashi, K. Usui, Y. Kazuta, T. Sunami, T. Matsuura, and T. Yomo. Darwinian evolution in a translation-coupled RNA replication system within a cell-like compartment. Nature Communications, 4(2494), 2013.

[4] T. Nakano, A. Eckford, and T. Haraguchi. Molecular Communication. Cambridge University Press, 2013. 